\title{
Redes sociales y consumo digital en jóvenes universitarios: economía de la atención y oligopolios de la comunicación en el siglo XXI
}

\author{
Social media use and digital consumption in university \\ students: the economy of attention and communication \\ oligopolies in the 21st century
}

\author{
Santiago Giraldo-Luque; Cristina Fernández-Rovira
}

Cómo citar este artículo:

Giraldo-Luque, Santiago; Fernández-Rovira, Cristina (2020). "Redes sociales y consumo digital en jóvenes universitarios: economía de la atención y oligopolios de la comunicación en el siglo XXI". Profesional de la información, v. 29, n. 5, e290528.

https://doi.org/10.3145/epi.2020.sep.28

Artículo recibido el 17-03-2020

Aceptación definitiva: 22-05-2020

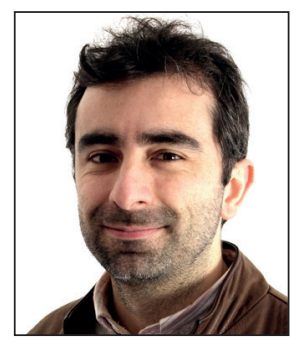
Santiago Giraldo-Luque $\triangle$ https://orcid.org/0000-0003-0024-7081
Universitat Autònoma de Barcelona Facultat de Ciències de la Comunicació Campus de la $U A B$ 08193 Bellaterra (Barcelona), España santiago.giraldo@uab.cat

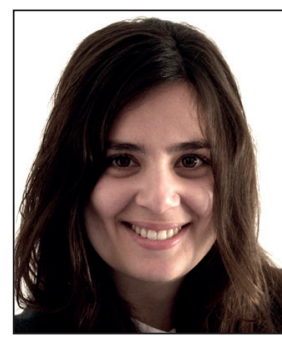

Cristina Fernández-Rovira https://orcid.org/0000-0003-0643-7329

Universitat de Vic - Universitat Central de Catalunya

Facultat d'Empresa i Comunicació

Sagrada Família, 7

08500 Vic (Barcelona), España

cristina.fernandez1@uvic.cat

\section{Resumen}

Se presenta el concepto de economía de la atención como centro del desarrollo de la industria comunicativa en la sociedad de la información, y se describen los resultados de un estudio empírico que demuestra la incidencia del concepto en la vida cotidiana de los estudiantes universitarios. La economía de la atención enmarca así una investigación que tiene el objetivo de identificar el tiempo de dedicación y las principales justificaciones que estudiantes universitarios de Comunicación en España dan a su uso de dispositivos móviles y de redes sociales. La investigación analiza la evolución de los resultados de una encuesta realizada en 2016, 2017, 2018 y 2019, en la que se pregunta sobre el uso y la percepción de la atención dedicada por ellos a las redes sociales. La encuesta se contrasta por una parte con una monitorización controlada del tiempo de uso de aplicaciones en los móviles efectuada con un grupo de estudiantes, y por otra con la realización de cuatro focus groups cualitativos. Los datos demuestran que los jóvenes analizados dedican cada vez más horas a las redes sociales y que además invierten más tiempo del que son conscientes en los dispositivos móviles, principalmente en dos redes-industrias que se convierten en oligopólicas: Instagram y WhatsApp. Asimismo, el estudio demuestra la dicotómica relación entre la consciencia de un uso adictivo e improductivo del tiempo en los móviles, y la propia necesidad de estar en estas plataformas. La discusión sobre la economía de la atención demuestra la eficacia y la interiorización individual de estrategias sofisticadas de control bajo la acción inconsciente del usuario.

\section{Palabras clave}

Economía de la atención; Redes sociales; Medios sociales; Estudiantes universitarios; Sociedad del control; Internet; Pantallas; Adicción; Teléfonos móviles; Oligopolios; Consumo digital; Instagram; WhatsApp.

\footnotetext{
Abstract

This article presents the concept of economy of attention as the center of development of the communication industry in the information society and describes the results of an empirical study that demonstrates the influence of this concept on university students' daily lives. This research using the economy of attention framework aims to identify the time dedicated as well as the justifications given by Spanish Communication university students to their use of mobile devices and social media. The research uses three complementary methods. Firstly, it analyzes the evolution of the results of a
} 
survey carried out in 2016, 2017, 2018, and 2019 in which university students were asked about their use and time spent on social media. The survey is confirmed against a controlled monitoring of the time of use of applications on mobiles carried out with a group of students and the realization of a series of four qualitative focus groups. The results show that university students spend more time on mobile devices than they are aware, concentrated on two platforms that have become oligopolistic industries: Instagram and WhatsApp. Likewise, the study reveals a dichotomous relationship between the awareness of an addictive and unproductive use of time on mobile screens, and the need to be on these platforms. A discussion based on the economy of attention shows the effect of sophisticated strategies effectively introduced as a social control mechanism via the users' unconscious actions.

\section{Keywords}

Economy of attention; Social media; Social networks; University students; Control society; Internet; Screens; Addiction; Smartphones; Oligopolies; Digital consumption; Instagram; WhatsApp.

\section{Introducción}

En el Reino Unido, según el Communications Market Report (Ofcom, 2018), los usuarios de teléfonos móviles de entre 18 y 24 años pasan un promedio de 211,56 minutos al día en sus smartphones, y el 65\% de los menores de 35 años consulta su teléfono en los primeros cinco minutos después de despertarse (Ofcom, 2018, p. 15). En Estados Unidos, el $48 \%$ de los jóvenes de entre 18 y 29 años manifiesta que nunca desconecta de su universo digital (Perrin; Kumar, 2019; Turkle, 2017). En España, las cifras recogidas por el broker de seguros dedicado al consumo, Rastreator (2019), indican que los jóvenes de 18 a 24 años pasan de media 366 minutos al día en sus teléfonos móviles, con un aumento del 12,6\% con respecto a las cifras de 2017. Además, según el Interactive Advertising Bureau, en España el 85\% de las personas usa redes sociales, de las que el $31 \%$ tiene una edad entre los 16 y los 30 años, y un $46 \%$ es universitario. Según el mismo estudio, los más jóvenes son los que más tiempo dedican a las plataformas sociales (IAB, 2019).

En cuanto a estudiantes universitarios, una investigación realizada en la State University of New York (Ahmed, 2019) demostró que más del $50 \%$ de los jóvenes observados declaraban utilizar su smartphone más de 9 horas al día. En China, una encuesta realizada a 337 estudiantes de universidades chinas halló que el 66,86\% de los participantes utilizaban el smartphone más de 4 horas cada día (Fu; Yin; Guo, 2019). De manera similar, los estudiantes de la Facultad de Negocios de la Universidad de La Salle Bajío (León, México), indicaron que su uso diario del móvil era de 7 horas (Amador et al., 2019). En España, el 31,5\% de los estudiantes de la Facultad de Ciencias de la Educación de la Universidad de Granada usan más de dos horas al día el teléfono móvil (Romero-Rodríguez; Aznar-Díaz, 2019), y más de cuatro horas al día los estudiantes de educación de la Universidad de Málaga y de la Universidad Autónoma de Madrid (Ruiz-Palmero et al., 2019, p. 196).

Los datos confirman un consumo adictivo del teléfono móvil en los jóvenes (Han; Kim, 2019; Rather; Rather, 2019; Abi-Jaoude, Treurnicht-Naylor; Pignatiello, 2020) que conduce a diversas patologías. A partir de los 15 años, según Gomes-Franco y Sendín-Gutiérrez (2014, p. 45), los jóvenes pueden empezar a desarrollar estrés o malestar ante la imposibilidad de conectarse a la Red. Otros estudios han corroborado alteraciones psicológicas tras sensaciones de:

- envidia social (Chou; Edge, 2012);

- perderse alguna actividad colectiva si no se accede al dispositivo (Przybylski et al., 2013);

- autoimagen negativa que se asocia a problemas de alimentación (Holland; Tiggemann, 2016);

- pensamientos suicidas debidos a cyberbullying (Sampasa-Kanyinga; Roumeliotis; Xu, 2014).

La concentración de la atención de los jóvenes en las pantallas determina además de las patologías anteriores, un nuevo campo de análisis orientado a la medición del consumo centrado en la atención digital y en las disputas entre las grandes plataformas digitales por captar la atención y los datos de los usuarios (Guillaud, 2018; Pasquinelli, 2009). El nuevo espacio de disputa se ha posicionado como el objeto de trabajo de la economía de la atención.

Las grandes compañías tecnológicas pivotan sobre la economía de la atención como centro de la economía del siglo XXI (Goldhaber, 2006; Morgans, 2017). El concepto describe los problemas de la sociedad de la información, en donde se construye un oligopolio estructurado sobre los bienes de la información, los datos (Mayer-Schönberger; Cukier, 2013; Franck, 2002), y el tiempo de atención consciente o inconsciente (Beller, 2006) que dedicamos a interactuar con las plataformas tecnológicas (Davenport; Beck, 2002; Roda, 2019). El tiempo es una buena medida para cuantificar el recurso que alimenta el propio sistema económico al introducir con cada clic, cada toque de pantalla o cada segundo de consumo, más datos.

La economía de la atención no es una novedad, pero en el momento en el que se comenzó a teorizar sobre ella en los años setenta, la tecnología aún no podía monitorizar perfectamente el comportamiento de los usuarios en su relación con las interfaces y tecnologías interconectadas (Davenport; Beck, 2002). La web 2.0 estaba aún lejos. Tampoco era posible hacer cálculos o algoritmos avanzados que fueran capaces de manejar la cantidad de información que los ordenadores comenzaron a almacenar ni tampoco eran capaces de realizar correlaciones entre ella (Shapiro; Varian, 1999, p. 36). 
La introducción en los años noventa de la web 1.0 y el comienzo de la masificación del uso de dispositivos conectados a la Red abrió posibilidades de análisis de la información consumida y del propio uso o comportamiento de los usuarios en internet. Pero no es hasta el fracaso de la primera internet y de su burbuja económica en 2000, cuando realmente se abre un nuevo rumbo en el que la creación de la información para la Web dejaría de ser un problema. Los usuarios empezaron a ser parte de plataformas en las que publicaban sus propios contenidos bajo el concepto de la web colaborativa y participativa, la web 2.0 (O’Reilly, 2007). El bien principal de la era de la información, que desplazaba la era industrial a partir de la introducción de un nuevo bien intangible, la información, tendía a ser producido a coste cero (Simon, 1971, p. 41; Davenport; Beck, 2002, p. 13; Fuchs, 2012).

El problema del siglo XXI no se concentra por tanto en la creación de la información, sino en la capacidad que tiene el producto para captar la atención del usuario. Según Shapiro y Varian (1999, p. 3), el precio de un bien de información está ligado no a su valor de producción, sino al propio valor de consumo. El producto como experiencia adquiere un valor específico cada vez que es consumido, no cuando es producido. La economía de la atención se centra en cuánto puede costar en términos de la escasez de atención, que alguien consuma la información producida y compartida. Davis y Meyer (1999) indican que el propio modelo de acumulación de capital se vincula también al producto inmaterial y por tanto resulta más importante la capacidad de crear atención sobre el producto que los propios costos de producción de los bienes.

El salto cualitativo que se produce con el avance tecnológico a partir de 2008 -pues las técnicas de recolección, extracción, captación, análisis y procesamiento de enormes cantidades de información resuelven los problemas de administración de los datos (Mayer-Schönberger; Cukier, 2013)- abre la puerta a la economía de la atención, un concepto que en la práctica concentra la oferta y la demanda de atención de una persona:

"cuanta más atención atraiga una plataforma, más efectiva es como espacio publicitario, lo que permite cobrar más a los que se quieren anunciar en la propia plataforma" (Morgans, 2017).

Este artículo presenta el concepto de economía de la atención y lo relaciona con el uso de las pantallas digitales y las redes sociales como espacios que concentran la atención de casi 4.500 millones de usuarios en el mundo (Hootsuite; We are social, 2019). El concepto permite desarrollar un estudio empírico efectuado con estudiantes de comunicación de entre 19 y 21 años de dos universidades españolas.
Se quiere ahondar en la discusión sobre la economía de la atención en una sociedad de control que se introduce de forma sofisticada, a través de la captación de los usuarios de internet en la vida cotidiana

Este trabajo responde bajo una metodología mixta a tres preguntas de investigación:

1. ¿Existe una brecha entre la atención real dedicada a las redes sociales y la percibida por los estudiantes universitarios analizados?

2. ¿En qué aplicaciones se concentra la atención de los estudiantes universitarios analizados en su uso del teléfono móvil?

3. ¿Cómo reaccionan los estudiantes universitarios analizados cuando conocen la atención real dedicada a las aplicaciones digitales, en especial, las redes sociales?

El artículo tiene el objetivo de ahondar en la discusión sobre la economía de la atención en el marco de una sociedad de control que se introduce de forma sofisticada, a través de la captación emotiva y psicológica de los usuarios de internet, en la vida cotidiana.

\section{Economía de la atención, un concepto clave}

La economía de la atención es un concepto teorizado por Goldhaber (1997) al hablar de la imposibilidad de prestar atención a toda la información que circula en internet. Davenport y Beck (2002, p. 32) proponen que la atención supone la concentración mental sobre una información específica que llega hasta los sentidos conscientes del individuo, quien decide, al prestarle atención, si debe realizar una acción. Los autores indican que

"la atención se produce en una fase de restricción relativamente inconsciente, en la que eliminamos la mayoría de los impulsos sensoriales que nos rodean y que fluyen hasta nosotros [...] y en una fase de decisión, en la que decidimos actuar respecto a la información a la que prestamos atención. Por tanto, entre la consciencia, la atención y la acción hay una relación de causalidad [...] la consciencia se transforma en atención cuando la información alcanza un umbral de significado en nuestro cerebro y desencadena el potencial de la acción" (Davenport; Beck, pp. 32-34).

Herbert Simon, uno de los primeros autores en enunciar el concepto de la economía de la atención, señalaba el trauma físico y mental que podía devenir de una sociedad tecnológica:

"una riqueza en información crea una pobreza en la atención y la necesidad de situar la atención -como bien escaso- de forma eficiente entre la sobreabundancia de fuentes de información que son factibles de consumir" (Simon, 1971, pp. 40-41). 
En la actualidad, una gran cantidad de estímulos de información compiten por los recursos cognitivos y dan lugar a la economía de la atención (Shapiro; Varian, 1999; Wise, 2012). La propia competencia por la atención escasa está determinada sobre todo por la prominencia que caracteriza a las élites tecnológicas (Franck, 2019a, 2019b; Fuchs, 2014) que pueden llegar a ser concentradoras o monopolizadoras de la atención.

El concepto, aún sin explotar tecnológicamente, avanza a finales del siglo XX con la introducción de los motores de búsqueda en la Web. Shapiro y Varian (1999, p. 21), al identificar la atención como un bien escaso en la economía de la información, describen los motores de búsqueda (y posteriormente los algoritmos de monitorización) como plataformas que permiten encontrar la información que las personas valoran y excluir la que no valoran. El cambio de formato a partir de la identificación de información personalizada da pie a:

- el reemplazo del intercambio comercial y comunicativo masivo y disperso para ingresar en el universo de la personalización del producto (Davenport; Beck, 2002, p. 27);

- la individualización del marketing;

- la observación comportamental de millones de consumidores.

Los tres procesos anteriores permiten desarrollar de forma inmediata y automatizada contenido especializado para cada usuario-cliente (Shapiro; Varian, 1999, p. 7).

Así, la economía de la atención funciona como cualquier otro recurso mercantil escaso y valioso:

- Se desenvuelve en un mercado en el que se compran y se venden los productos clave bajo leyes de oferta y demanda.

- El producto o bien principal es escaso y tiene límites perfectamente definidos.

- La mercancía es perecedera: si se pierde es altamente probable que no pueda recuperarse.

- Implica un incremento de los beneficios ligado a la prominencia de los actores relevantes:

"cuanta más atención se tiene, más fácil es conseguir aún más atención” (Davenport; Beck, 2002, p. 24).

Como escribe Giffard (2013), en el contexto de la economía de la atención, el tiempo se convierte en una variable determinante y ello hace que en las industrias culturales se libre una batalla por la captación de tiempo libre. Dentro de internet, las redes sociales son el escenario privilegiado para el mercado de la atención y el lugar en el que puede producirse con mayor facilidad la concentración de la atención del usuario. Ciampaglia, Flammini y Menczer (2015) estudian a nivel agregado el fenómeno de la economía de la atención, y se centran en las pautas de la atención colectiva que se relacionan con la información nueva. Para los autores, la producción de un nuevo conocimiento está asociada a cambios significativos de atención colectiva, lo que es consistente con un escenario en el que la asignación de atención hacia un tema o plataforma estimula la demanda de información al respecto y, a su vez, el suministro de información novedosa -a coste cero- adicional. Es el lugar del capitalismo cognitivo, en el que se especula con el conocimiento, pero también con las capacidades psíquicas, los sentimientos y la atención (Guillaud, 2018).

Para Tristan Harris, quien trabajó para Google como diseñador ético y actualmente es el director y co-fundador del Centre for Humane Technology, la atención se rige por "recompensas variables intermitentes" (Morgans, 2017). Ello conduce a que sea posible el control de la atención por parte de las compañías tecnológicas dueñas de las principales redes sociales (Fuchs, 2018; Sunstein, 2017) que, a través de tecnologías llamativas asociadas a las experiencias emotivas del usuario como el botón "me gusta", determinan un mercado de atracción muy eficaz (Gerlitz; Helmond, 2013). La nueva relación con la tecnología, a la que se dedica una dosis importante de atención bajo un bucle permanente de notificaciones, incide además sobre el control de la propia atención que determina el dominio de la experiencia-acción del individuo e influye en su calidad de vida (Csikszentmihalyi, 1997).

En esa misma línea Justin Rosenstein, creador del botón "me gusta” de Facebook, advertía que

"la mayoría de las notificaciones instantáneas son sólo distracciones que nos sacan del presente. Nos obligan a sacar el móvil y a perdernos en una descarga de información que podría esperar para más tarde o que ni siquiera es relevante" (Rosenstein en Morgans, 2017).

De igual forma, James Williams, ex estratega de Google, señalaba que la industria tecnológica de la información es la "más larga, más estandarizada y más centralizada forma de control de la atención en la historia de la humanidad" (Williams en Lewis, 2017).

Para Williams, el marco de la economía de la atención también se autoalimenta de la creación y de la impulsividad:

"La economía de la atención incentiva el diseño de tecnologías que llaman nuestra atención. Al hacerlo, privilegia nuestros impulsos sobre nuestras intenciones" (Williams en Lewis, 2017).

Una situación que ha obligado también a los medios de comunicación a sumarse a la oferta informativa impulsiva, sensacionalista: "cebos -baits-y entretenimiento para sobrevivir" (Williams, en Lewis, 2017). 
Rahwan (2018) argumenta que los avances de la inteligencia artificial han hecho aflorar preguntas acerca de cómo regular los sistemas algorítmicos complejos que determinan y preparan las notificaciones que llaman nuestra atención y propone combinar el Human in the loop con un nuevo contrato social que tenga en cuenta los algoritmos. El autor denuncia que los algoritmos son cajas negras (Pasquale, 2015), que determinan el comportamiento de atención de miles de millones de individuos, una idea que asociada a la economía de la atención, ha sido profundizada por Celis-Bueno (2017). Las cuestiones sobre los algoritmos también se relacionan con las burbujas informativas (Pariser, 2011) y con los sistemas de apoyo de decisiones construidos a través de los datos. Los nuevos tipos de orientación de las selecciones, como forma de poder comunicativo (Luhmann, 1995), pueden perpetuar la injusticia social pues son sesgados desde su diseño o porque aprenden de los propios sesgos humanos (Caliskan; Bryson; Narayanan, 2017).

\section{Metodología}

Esta investigación, que propone la economía de la atención como el sustento teórico de la configuración de monopolios dentro de la sociedad de la información y del modelo de capitalismo cognitivo (Guillaud, 2018; Pasquinelli, 2009), establece el control y la medida del tiempo de uso del teléfono móvil y de las redes sociales como la medida principal para determinar la atención dedicada (Simon, 1971; Davenport; Beck, 2002) al dispositivo y a las aplicaciones de redes sociales por parte de los usuarios analizados.

Los métodos de investigación usados se estructuran en tres dimensiones:

- en primer lugar, se utiliza una encuesta que mide la autodeclaración del tiempo de uso que los estudiantes observados emplean en las redes sociales;

- en segundo lugar, se realiza una monitorización de la actividad de una muestra de la población estudiada en sus teléfonos móviles para evidenciar el tiempo real de uso del dispositivo, con especial énfasis en el tiempo invertido en el consumo de redes sociales;

- por último, se llevan a cabo cuatro focus group con los estudiantes participantes, con la intención de indagar sobre las justificaciones del propio uso de las aplicaciones móviles y, principalmente, de los medios sociales.

En los tres métodos la muestra de estudio está compuesta por estudiantes universitarios, de entre 19 y 21 años, que cursan el segundo o el tercer año de estudios de grado relacionados con las ciencias de la comunicación (Periodismo, Comunicación Audiovisual o Publicidad y Relaciones Públicas), en la Universitat Autònoma de Barcelona (UAB) y en la Universitat de Vic-Universitat Central de Catalunya (UVic-UCC).

La elección de la muestra se justifica a partir de estudios precedentes en los que se analizó el público de edades similares (Ofcom, 2018; Perrin; Kumar, 2019; Turkle, 2017) y, más concretamente, investigaciones con estudiantes universitarios en diversos países, como los Estados Unidos (Ahmed, 2019); China (Fu; Ying; Guo, 2019); México (Amador et al., 2019) y España (Romero-Rodríguez; Aznar-Díaz, 2019; Ruiz-Palmero et al., 2019). El hecho de centrar la investigación en estudiantes de Comunicación es interesante en cuanto a elemento de contraste con otras titulaciones, sobre todo para futuros estudios, en tanto que a los estudiantes de comunicación se les presupone un conocimiento del ramo mayor que a los de otras especialidades.

La encuesta se presenta como un método adecuado de recolección de datos cuantitativos para conocer el tiempo dedicado al uso del móvil y a las redes sociales en la muestra seleccionada. Estudios previos han utilizado la encuesta con un diseño transversal para medir el tiempo y la caracterización de uso del móvil y de las redes sociales en estudiantes universitarios (Carcelén; Mera; Irisarri, 2019; Fu; Ying; Guo, 2019; Romero-Rodríguez; Aznar-Díaz, 2019). En el presente estudio, a diferencia de investigaciones previas, las oleadas de encuesta realizadas presentan una aproximación longitudinal de tendencia (Del-Val-Cid, 2015, p. 126), ya que el cuestionario se ha aplicado en 2016, 2017, 2018 y 2019 , lo que permite observar la evolución de los datos de consumo en una muestra de la población universitaria con características comunes (tabla 1). En este caso, se utilizan los resultados de la pregunta: “¿Cuánto tiempo aproximado dedicas al día a navegar por las redes sociales?" del estudio de uso y caracterización de los usuarios de redes sociales en estudiantes de comunicación realizado por Tejedor, Carniel-Bugs y Giraldo-Luque (2019) y Giraldo-Luque, Tejedor y Carniel-Bugs (2017). La pregunta permite analizar de forma descriptiva dos variables específicas:

- tiempo dedicado a las redes sociales por los estudiantes en cada uno de los cuatro años analizados;

- evolución de la muestra longitudinal de tendencia sobre los mismos datos de uso de redes sociales.

La muestra de estudio utilizada para las encuestas está formada por un promedio de 238 estudiantes universitarios por año de estudio, con la caracterización por año de muestreo que se observa en la tabla 1.

Tabla 1. Muestra de encuestas por año de estudio

\begin{tabular}{|c|c|c|c|c|c|}
\hline \multirow{2}{*}{ Año } & \multirow{2}{*}{$\begin{array}{c}\text { Número de encues- } \\
\text { tas }\end{array}$} & $\begin{array}{c}\text { Edad media de } \\
\text { encuestados }\end{array}$ & \multicolumn{2}{|c|}{$\begin{array}{c}\text { Porcentaje de mujeres y hombres } \\
\text { Hombres }\end{array}$} \\
\hline 2016 & 215 & 20,3 & Mujeres & 35,6 \\
\hline 2017 & 280 & 20,3 & 64,4 & 41,0 \\
\hline 2018 & 168 & 19,5 & 59,0 & Enero de 2016 \\
\hline 2019 & 288 & 19,7 & 64,9 & 35,1 \\
\hline
\end{tabular}


El método aplicado permite contrastar los datos de la encuesta con dos técnicas más:

- monitorización del tiempo de uso del móvil;

- desarrollo de una serie de grupos focales cualitativos.

Así, se propone dar un paso más allá de estudios que o bien sólo utilizan la encuesta como método, o se centran en la monitorización de usuarios a través de aplicaciones específicas. Los grupos focales y el ejercicio de monitorización fueron diseñados bajo un muestreo sistemático (Mays; Pope, 1995) que permitió identificar los perfiles típicos para el análisis: los estudiantes de Comunicación de la Universitat Autònoma de Barcelona y la Universitat de Vic-Universitat Central de Catalunya.

El ejercicio de monitorización implementado para medir el tiempo real y, por tanto, la atención vertida sobre el dispositivo móvil y sus aplicaciones consistió en la observación de un grupo de 25 estudiantes universitarios de Comunicación elegidos aleatoriamente. Asimismo, para los grupos focales se convocó a los mismos estudiantes. Las características demográficas del grupo fueron:

- sexo: $64 \%$ mujeres y $36 \%$ hombres;

- edad: entre 19 y 21 años;

- grado universitario: 52\% estudiantes de Periodismo; 36\% de Comunicación Audiovisual; 12\% de Publicidad y Relaciones Públicas).

Para la prueba de monitorización, cada participante controló el uso de su teléfono móvil durante una semana (entre octubre y noviembre de 2019) a través de la aplicación Quality Time. En la línea de estudios precedentes, que han hecho pruebas similares durante el mismo período de tiempo (Vempati; Bhuma; Fiaidhi, 2020; Gower; Moreno, 2018; Deng et al., 2018), el seguimiento permitió conseguir datos reales de tiempo de uso y observar qué aplicaciones son las que concentran la atención de los usuarios. Las variables analizadas a partir de los datos obtenidos por Quality Time fueron:

- tiempo de uso del teléfono móvil total (en horas y minutos a la semana);

- las 6 aplicaciones a las que más atención se prestó durante la semana por cada participante;

- atención vertida en cada aplicación durante la semana de estudio.

La monitorización para la obtención de datos se complementa con el desarrollo de cuatro focus groups de entre seis y siete estudiantes universitarios de Comunicación, con una duración aproximada de 60 minutos cada uno, realizados entre el 2 y el 13 de diciembre de 2019.

En los grupos focales se discutieron tres temas principales mediante preguntas abiertas. Éstos fueron posteriormente categorizados para su análisis:

- percepción de cada usuario de la atención dedicada al móvil y, en especial, a las redes sociales en términos de tiempo;

- resultados reales de la atención dedicada en los smartphones y las redes sociales;

- justificación y motivaciones de cada participante para explicar la alta cantidad de atención dedicada al móvil y a las redes sociales.

\section{Resultados}

\subsection{Los estudiantes universitarios confiesan su adicción a las redes sociales}

Los datos obtenidos en la encuesta revelan que existe una tendencia al alza en el número de horas al día que los jóvenes encuestados dicen usar las redes sociales. El aumento se concentra particularmente en el año 2019. En 2016, el 28,8\% de los preguntados manifestaba usar las redes sociales entre 2 y 4 horas al día, mientras que en 2017 era el 37,5\% y, en 2018, el 41\%. Los datos de 2019 son aún más reveladores porque si bien baja el porcentaje de jóvenes estudiantes de las áreas de Comunicación en las universidades analizadas que usan las redes entre 2 y 4 horas (24,6\%), aumenta de forma considerable el porcentaje de encuestados que les dedica entre 4 y 6 horas al día. Si para 2016 el porcentaje era de 9,3\%, para 2019 es del 23,6\%. En una lectura similar, aquellos que decían usar las redes entre 1 y 2 horas al día han disminuido en función del aumento de otras franjas de más consumo. Si para 2016 eran el 32\% de la muestra, para 2017 fueron el $30 \%$, para 2018 el $32 \%$ y para 2019 se redujeron a sólo el 15\%. Estos resultados principales están reflejados en el gráfico 1.

\begin{tabular}{|c|c|c|c|c|c|c|c|c|}
\hline & $\begin{array}{l}\text { Menos de } 1 \\
\text { vez al día }\end{array}$ & $\begin{array}{l}\text { Menos de } 1 \\
\text { hora al día }\end{array}$ & $\begin{array}{l}\text { Entre } 1 \text { y } 2 \\
\text { horas al día }\end{array}$ & $\begin{array}{l}\text { Entre } 2 \text { y } 4 \\
\text { horas al día }\end{array}$ & $\begin{array}{l}\text { Entre } 4 \text { y } 6 \\
\text { horas al día }\end{array}$ & $\begin{array}{l}\text { Entre } 6 \text { y } 8 \\
\text { horas al día }\end{array}$ & $\begin{array}{l}\text { Entre } 8 \text { y } 10 \\
\text { horas al día }\end{array}$ & $\begin{array}{l}\text { Más de } 10 \\
\text { horas al día }\end{array}$ \\
\hline 2016 & $0.9 \%$ & $19.1 \%$ & $32.1 \%$ & $28.8 \%$ & $9.3 \%$ & $2.8 \%$ & $2.3 \%$ & $4.7 \%$ \\
\hline 2017 & $1.4 \%$ & $6.4 \%$ & $30.0 \%$ & $37.5 \%$ & $16.8 \%$ & $4.6 \%$ & $1.4 \%$ & $1.8 \%$ \\
\hline 2018 & $2.4 \%$ & $5.4 \%$ & $32.7 \%$ & $41.1 \%$ & $12.5 \%$ & $2.4 \%$ & $3.0 \%$ & $0.6 \%$ \\
\hline 2019 & $1.4 \%$ & $3.1 \%$ & $5.2 \%$ & $24.7 \%$ & $23.6 \%$ & $21.5 \%$ & $13.9 \%$ & $6.6 \%$ \\
\hline
\end{tabular}

Grafico 1. Tiempo de atención diario dedicado al uso de redes sociales (2016-2019). Los datos del gráfico indican el tiempo aproximado de uso de redes sociales de la UAB y UVIc-UCC encuestados entre 2016 y 2019 (autodeclaración). 
De acuerdo con la tendencia al alza en el tiempo empleado en las redes sociales, se observa que una minoría de encuestados las usa menos de una vez al día. Por último, los datos denotan un importante incremento en el uso de las redes durante muchas horas. Es destacable que los jóvenes que usan las redes entre 6 y 8 horas al día pasan a ser el 21,5\% en 2019, cuando entre 2016 y 2018 apenas llegaban al 5\%. Asimismo, el porcentaje de estudiantes que utiliza las redes más de 8 horas al día también se multiplica. Entre 2016 y 2018 los encuestados que más usaban las redes no superaban el 7\%, mientras que en 2019 la cifra se eleva hasta el $20,5 \%$.

Los propios estudiantes reconocieron en los focus groups que:

"puedo estar cada cinco minutos mirando si hay alguna novedad en el Instagram" (estudiante 11).

al tiempo que señalan que

"aunque no es una actividad constante e ininterrumpida, suelo estar entre dos y cinco horas en las redes sociales, principalmente en Instagram" (estudiante 7).

También están de acuerdo en que

"a veces entras a las redes por una cosa y acabas haciendo muchas otras en ellas sin darte cuenta, pierdes el sentido del tiempo..., somos adictos y vamos a ser más dependientes, cada vez lo seremos más".

Asimismo, los estudiantes observados agregan que acceder a las redes sociales a través del móvil es ya "una actividad rutinaria" (estudiantes 3 y 8). Otro de los jóvenes participantes en los grupos de discusión explicaba:

"A veces ni siquiera te enteras de lo que ves, pero sientes la necesidad de estar ahí, en la pantalla, haciendo scroll, sin más".

Los estudiantes también declararon en el focus group que

"puedo estar cuando me despierto más de una hora con el móvil en la cama, mirando las redes, simplemente se me va el tiempo" (estudiante 22).

Una declaración que conecta con su propia sorpresa al comprobar el tiempo real que pasa en sus dispositivos móviles y en las redes sociales después de la lectura de datos del ejercicio de monitorización:

"hago 6 horas en redes sociales al día, y media hora en aplicaciones de productividad, ies muy fuerte! Me da miedo, qué fuerte..." (estudiante 23).

Otro estudiante también se sorprendía:

“pensaba que era mucho menos..., madre mía” (estudiante 15).

Es el mismo caso cuando expresan que están

"siempre conectados. Es que así no lo mires, el teléfono te manda cosas por sí solo, no puedes evitar prestarle atención" (estudiante 4).

A pesar de ello, los estudiantes, en su autodeclaración de uso, son conscientes de que el tiempo empleado en las redes sociales es "excesivo".

\subsection{Control del tiempo real, una preocupación (momentánea)}

La monitorización del tiempo real de consumo realizada pone de manifiesto que, en promedio, los universitarios estudiados usan el móvil 1.863,5 minutos a la semana, es decir, 31 horas. Los datos arrojan un consumo diario de 266,2 minutos (4 horas y 26 minutos). Los resultados indican un consumo de 54,7 minutos más de lo que declararon los usuarios jóvenes en el Reino Unido (Ofcom, 2018), aunque menos de lo que declararon los universitarios estadounidenses (Ahmed, 2019) y mexicanos (Amador et al., 2019) y los jóvenes en general en España (Rastreator, 2019). Aunque las métricas comparadas con los estudios universitarios son difíciles de hacer debido a que las escalas de medida utilizadas en cada estudio son diferentes, el gráfico 2 traza un marco comparativo de la encuesta aplicada con los estudios similares realizados en España (Universidad de Málaga, Universidad Autónoma de Madrid, Universidad de Granada), China (varias universidades) y Estados Unidos (State University of New York).

De las horas semanales monitorizadas, los jóvenes destinan de media 7 horas y 21 minutos a WhatsApp (un poco más de una hora al día) y más de 10 horas a Instagram (casi una hora y media cada día). La información extraída también indica que las cuatro aplicaciones más usadas en el teléfono móvil son redes sociales:

- WhatsApp, utilizada por el 96\% de los usuarios estudiados;

- Instagram, 84\%;

- YouTube, 80\%;

- Twitter, 52\%. 


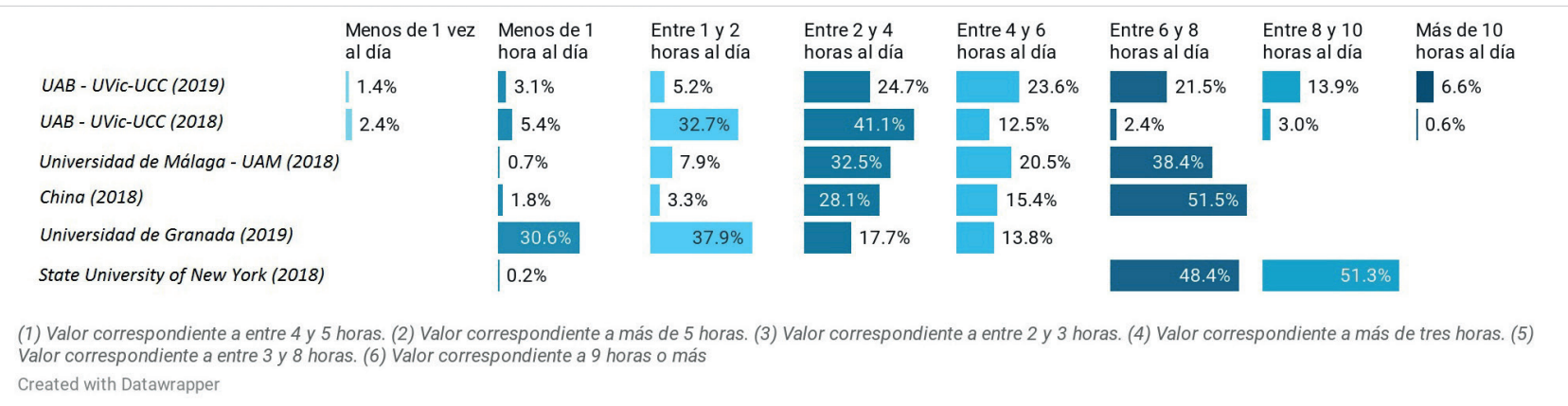

Grafico 2. Comparativa de estudios sobre el tiempo de uso del móvil en estudiantes universitarios. Los datos del gráfico realizan una comparación entre 6 estudios llevados a cabo entre 2018 y 2019 en diferentes universidades. Todos los estudios recopilan información de estudiantes universitarios mediante el uso de encuestas.

La tabla 2 muestra las seis aplicaciones más utilizadas y el promedio de tiempo de uso entre los 25 usuarios participantes en la monitorización.

Tabla 2. Aplicaciones más usadas por los jóvenes (porcentaje de usuarios y promedio de tiempo de uso)

\begin{tabular}{|l|c|c|l|l|}
\hline \multicolumn{1}{|c|}{ Aplicación } & $\begin{array}{c}\text { Porcentaje de } \\
\text { usuarios }\end{array}$ & $\begin{array}{c}\text { Promedio de tiempo de uso } \\
\text { semanal (minutos) }\end{array}$ & $\begin{array}{c}\text { Promedio de tiempo de uso } \\
\text { semanal (horas y minutos) }\end{array}$ & $\begin{array}{c}\text { Promedio de uso diario } \\
\text { (horas y minutos) }\end{array}$ \\
\hline WhatsApp & 96 & 441,3 & 7 horas, 21 minutos & 1 hora, 3 minutos \\
\hline Instagram & 84 & 608,8 & 10 horas, 8 minutos & 1 horas, 27 minutos \\
\hline YouTube & 80 & 344,3 & 5 horas, 44 minutos & 49 minutos \\
\hline Twitter & 52 & 233,1 & 2 horas, 53 minutos 3 minutos & 33 minutos \\
\hline Chrome & 48 & 123,1 & 6 horas, 28 minutos & 55 minutos \\
\hline Netflix & 28 & 388,0 & & \\
\hline
\end{tabular}

Los resultados de la tabla 2 indican una concentración de la atención en cinco aplicaciones. El porcentaje de uso de las principales redes sociales en el móvil (WhatsApp, Instagram, YouTube y Twitter) ocupa el 84,5\% del tiempo de uso de los teléfonos inteligentes. Los datos hacen evidente el dominio de WhatsApp y de Instagram (pertenecientes a la misma compañía, Facebook Inc.), tanto en el porcentaje de usuarios que las utilizan, como en el tiempo de uso de los dispositivos móviles dedicado a ellas. En el caso de Instagram, la aplicación que más concentra la atención de los usuarios, el porcentaje del tiempo dedicado a la red social sobre el total de uso del dispositivo es de un tercio (33\%) (ver gráfico 3).

Instagram es casi omnipresente en las declaraciones de uso del tiempo de los estudiantes. Algunos estudiantes remarcaban:

"a veces sientes la necesidad de estar allí, de entrar a Instagram y ver las fotos, aunque las hayas visto cientos de veces. Es una necesidad".

Los jóvenes participantes en los grupos focales también declaraban:

"Gracias al Insta podemos hablar con gente que está lejos o que no ves hace tiempo, pongo una foto y dicen: ‘¿estás de viaje?’ y entonces hablamos un poco, de otra manera no hablaríamos nunca... También, puedo contactar con personas con las que hace años no me veo y cuando comento alguna de sus stories puedo tener alguna posibilidad de quedar con ella, es algo que me ha pasado muchas veces..., si no los tuviera en el Insta, adiós muy buenas... (estudiante 2).

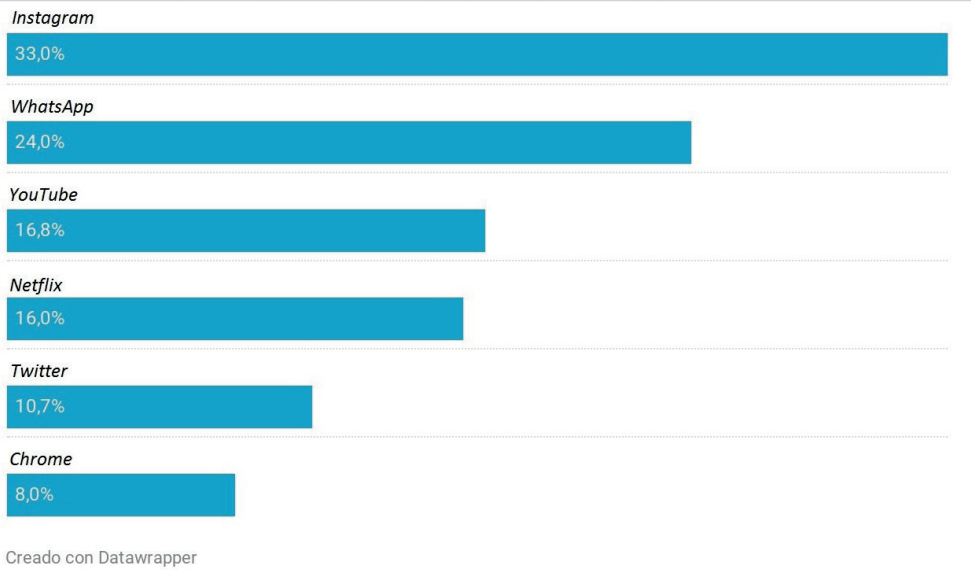

Grafico 3. Porcentaje de tiempo dedicado a las aplicaciones (total de usuarios). El gráfico indica el promedio general del tiempo dedicado por los jóvenes universitarios monitorizados a cada aplicación (las 6 más utilizadas ) sobre el total del tiempo de uso del móvil a la semana. El promedio corresponde a toda la muestra observada $(n=25)$. 
A pesar de que los estudiantes reconocieron que su consumo era excesivo e incluso adictivo, la mayoría de ellos no ha pensado dejar las redes sociales:

"Yo creo que nosotros hemos crecido con ellas y no las dejamos por eso, porque siempre han estado allí, con nosotros. A veces pienso, y cómo van a contactar conmigo si no estoy en las redes..., bueno, puede ser que me llamen, pero si estoy en Insta, será más fácil (estudiante 8).

Dentro del focus group la mayoría de los estudiantes aseguraron que ni siquiera dejarían las redes para siempre por una suma de dinero (entre 1.000 y 5.000 euros). De hecho, la opinión general manifestada por acuerdo en los ejercicios de conversación y que justifica la imposibilidad de dejar de usar las redes de forma excesiva es que sienten un fuerte vínculo con ellas y con lo que pasa en estas plataformas en relación con su sociabilidad. Los estudiantes participantes hacen evidente sus impedimentos emotivos para no alejarse de ellas. Al respecto, indican:

"Siempre tienes la necesidad de estar conectado..., saber qué está pasando. Es como una necesidad vital. En las redes sociales te sientes como si fueras parte de algo, si te vas de ellas es como si dejaras de ser parte de ello" (estudiante 10).

Es una idea reafirmada por otros participantes:

"Nos han creado un cierto miedo. Nos sentimos solos si estamos fuera de las redes, y más si estamos sin el teléfono móvil. Creo que todo el mundo tiene miedo a no ser nadie" (estudiante 14).

Una percepción que se reafirma con la propia experiencia vivida por uno de ellos:

"El otro día tuve que dejar el teléfono móvil en casa porque estaba sin batería y, cuando salí a la calle, tenía miedo..., me podría pasar algo" (estudiante 20).

En la discusión se hace evidente también la paradoja del consumo excesivo que se hace consciente para los estudiantes. A pesar de que dicen que no podrán dejar de usar las redes por los vínculos emotivos y las necesidades indicadas, los propios resultados del focus group manifiestan sin embargo una reflexión crítica ante un uso poco controlado de las redes sociales:

"Es muy importante el tiempo que gastamos en las redes, porque, además, es un tiempo ligado al entretenimiento. Gastamos mucho tiempo en entretenimiento cuando podríamos estar haciendo cosas mucho más productivas o que llevaran a algo real" (estudiante 19).

La preocupación de los estudiantes participantes se ilustra como momentánea porque, a pesar de reconocer el uso excesivo, los jóvenes participantes no están dispuestos a dejar de usar las redes sociales. La implicación emotiva que tienen con las redes es apreciada como una necesidad vital, ahora ligada al universo comunicativo. Diversos autores (Turkle, 2017; Celis-Bueno, 2017; Morgans, 2017; Franck, 2019b) llaman la atención sobre la capacidad de las redes para inducir ese consumo adictivo relacionado con la creación de una necesidad social (emocional) que se ha hecho común y que se ha terminado por aceptar, particularmente en los jóvenes.

\section{Discusión de resultados}

La necesidad de seleccionar a qué insumo informativo procedente de internet se presta atención es un hecho constatable, que sigue la línea de los estudios de Simon (1971); Goldhaber (1997); Shapiro y Varian (1999) y Davenport y Beck (2002), en el contexto del capitalismo cognitivo (Guillaud, 2018). Como se ha comprobado en la investigación, la tendencia al alza en el número de horas al día dedicadas a las redes sociales convierte ese tiempo en una valiosa mercancía (Morgans, 2017) para las empresas propietarias de las redes sociales, que compiten como nuevas industrias culturales (Giffard, 2013) por la captación de la atención.

En términos comparados, como se señaló en el trabajo de Ruiz-Palmero et al. (2019), el estudio ratifica que los universitarios españoles pasan más de cuatro horas al día en sus smartphones y que el consumo de los estudiantes analizados de los grados de Comunicación de la Universitat Autònoma de Barcelona (UAB) y la Universitat de Vic-Universitat Central de Catalunya es similar al de los estudiantes de Educación en la Universidad de Málaga y la Universidad Autónoma de Madrid. Aun así, a pesar de ser números muy elevados, son menores que los identificados en los estudiantes de México (Amador et al., 2019) y que la mayoría de los estudiantes de la State University of New York (Ahmed, 2019). Llama la atención la diferencia particular de los datos con el estudio de Romero-Rodríguez y Aznar-Díaz (2019, p. 4), realizado con estudiantes de Educación de la Universidad de Granada, quienes identificaron un muy alto porcentaje de estudiantes $(68,5 \%)$ que usa el smartphone menos de dos horas al día.

Los tiempos de atención detectados en el consumo de redes sociales confirman el propio universo hiperconectado e insertado en la individualidad cotidiana de los usuarios participantes del estudio, como lo documentaron previamente los informes de Dscout's (2016) y Hootsuite y We are social (2019).
Los usuarios estudiados usan el móvil $1.863,5$ minutos a la semana, es decir 31 horas, un consumo diario de 4 horas y 26 minutos 
El estudio constata también el creciente tiempo de uso de las redes sociales $y$, al mismo tiempo, pone de relieve una relativa inconsciencia del usuario (Beller, 2006) sobre su propio uso y de cómo los recolectores de datos logran captar su atención:

"La vitalidad, las luces, los colores, todo es Ilamativo, nos atrae, lo tenemos todo en ese lugar, en el móvil y, sobre todo en las redes sociales..., allí todo es más fácil, todo está dentro de estas plataformas" (estudiante 5).

Al mismo tiempo, las acciones que realizan los jóvenes en las redes a través de sus smartphones son, como se desprende de las observaciones de los propios estudiantes, muchas veces involuntarias o inconscientes:

"Yo uso Instagram, pero no para colgar fotos, sino para mirar constantemente lo que me interesa..., bueno (confiesa el estudiante después de una corta pausa), creo que sí, es un uso adictivo" (estudiante 16).

Así, la correlación planteada por Davenport y Beck (2002) entre la consciencia de la atención como reacción a un estímulo informativo y que promueve una acción o una toma de decisión, es llevada al extremo bajo el manto de la emotividad generada por la propia atención humana que promueven las notificaciones de las redes sociales. La propia inconsciencia de la acción de mirar el móvil es en sí misma una forma de canalización de la atención mucho más sofisticada e interiorizada dentro del propio mecanismo de funcionamiento neuronal y emocional del ser humano (Fuchs, 2018; Sunstein, 2017):

“A veces cuando quería estudiar debía guardar el móvil en un cajón para poder concentrarme, no sé, igual acababa mirándolo de vez en cuando porque tenía la sensación de que podía perderme algo importante"

"Yo, incluso, a veces dejo el móvil aislado, o apagado, pero tengo la esperanza o la ilusión de que cuando lo encienda, alguien me haya enviado un mensaje, que alguien me necesite...".

La mirada constante al dispositivo se presenta como una elección no libre porque nace, en muchas ocasiones, del automatismo emotivo ligado a la necesidad social de tener algo que ver en la pantalla, de sentir que alguien nos envía un mensaje, nos da un like, o nos escribe un comentario (Turkle, 2017; Luhmann, 1995). Dos estudiantes comentaban al respecto:

"Yo hace como dos años que no cuelgo fotos porque siento una presión personal de saber cuánta gente la verá. Es patético, pero es así, tengo la presión de que la gente la vea, la comente"

"Si yo pongo una foto y nadie me da un like..., bueno, sería horrible".

El individuo desindividualizado o desvirtualizado (Giraldo-Luque, 2015) termina por caer, programado emocionalmente, en los cebos -baits-definidos por los propios algoritmos sofisticados que se nutren del contenido y de las interacciones que el propio usuario comparte y realiza en sus dispositivos y en sus perfiles de las redes sociales, aunque casi siempre sean distracciones intrascendentes (Rosenstein en Morgans, 2017).
"Siempre tienes la necesidad de estar conectado..., saber qué está pasando. Es como una necesidad vital. En las redes sociales te sientes como si fueras parte de algo, si te vas es como si dejaras de ser parte de ello" (estudiante 10)

El control sofisticado de la acción del usuario (Žižek, 2018) se materializa al provocar una distracción que es más potente que cualquier otro impulso de atención y que actúa como una recompensa variable intermitente (Harris en Morgans, 2017) que influye en la calidad de vida del usuario (Csikszentmihalyi, 1997) y en sus decisiones, tal y como se observa en el estudio al comprobar que los estudiantes participantes usan las redes sociales para fines distintos de los que tenían previstos inicialmente. La sofisticación del control (Marcuse, 1987) acaba por garantizar la atención del usuario sobre el smartphone y las aplicaciones sociales.

Lo más interesante -y tenebroso- del juego perfecto de los sistemas avanzados de control tecnológico (Wise, 2012) es que responde a dos ideas futuristas presentadas en la prehistoria de la economía de la atención. En primer lugar, la monitorización que guía las preferencias de los usuarios era antes consciente. Davenport y Beck reclamaban a comienzos de siglo:

“ni qué decir tiene que la medición de la atención puede ser intrusiva. Hasta la fecha, quienes han medido la atención lo han hecho con el consentimiento expreso de los medidos. Esperamos y deseamos que siga siendo así" (Davenport; Beck, 2002, p. 68).

Davenport y Beck introducían también el segundo hito tecnológico del futuro:

"el proveedor informativo querrá saber quién está prestando atención a los mensajes y alguien tendrá que poner a su disposición una tecnología de medición de la atención” (Davenport; Beck, 2002, p. 67). 
La tecnología del algoritmo y la capacidad de las máquinas para almacenar y procesar información propició los potenciales elementos de control sobre la actividad del individuo y que permiten medir el comportamiento de la atención cotidiana en internet.

\section{El individuo cae en los cebos definidos por los algoritmos sofisticados, que casi siem- pre son distracciones intrascendentes}

Además de las dos ideas anteriores, el tercer elemento que permite el desarrollo de la economía de la atención está significado en la propia retroalimentación sistémica de la tecnología y de los insumos del movimiento económico de lo intangible (Mayer-Schönberger; Cukier, 2013). Antes de forma consciente y autorizada, pero ahora a través de un proceso automatizado e invisible para el propio usuario controlado -y espiado-, el individuo envía nuevos datos de consumo y de su propio comportamiento digital al recolector oligopolista de la información, a coste cero (Celis-Bueno, 2017).

La concentración de tal distracción y de la atención genera más datos para ajustar las cajas negras o algoritmos que determinan los intereses del consumidor (Rahwan, 2018) y, al mismo tiempo, permiten un mayor y más refinado control sobre el usuario (Celis-Bueno, 2017; Franck, 2019a). Por eso resulta vital que el usuario piense, como alguno de los participantes, que sus datos e información, individual, no son relevantes:

"no entiendo por qué tienen tantos datos míos si, en realidad, mis datos personales no son relevantes, no son valiosos".

La predominancia de número de horas dedicadas a las redes sociales ratifica el oligopolio de la economía de atención en la sociedad digital, casi siempre convertida en imagen (Instagram). Los datos están estrechamente relacionados con el tipo y construcción de la sociedad de control y con las estrategias de las redes sociales para captar y mantener la atención del usuario-consumidor (Williams en Lewis, 2017), y también al concepto de prominencia (Franck, 2019b) ligado a WhatsApp e Instagram, constituidas como élites recaudadoras de datos que responden al modelo de economía de la atención. El comportamiento económico de las dos redes describe a la perfección el incremento de los beneficios bajo un sistema de capital que tiende a ser monopólico:

“cuanta más atención se tiene, más fácil es conseguir aún más atención” (Davenport; Beck, 2002, p. 24).

Según los resultados del estudio, WhatsApp e Instagram concentran la mayoría de la atención, lo que refuerza el poder de agencia de determinados nodos de internet (Fuchs, 2014), como paradoja al imaginario utopista o efecto placebo (Giraldo-Luque, 2015) en el que se cree que todos tienen la misma posibilidad de captación de la atención en la Red.

\section{Conclusión: la necesidad social de estar conectados}

En la economía de la atención, la información disponible y concentrada en pocos gestores de datos (oligopolios) convierte la propia información de coste cero (producida por los usuarios) en un bien de exclusión de información para la toma de decisiones en todos los campos de la vida y con un elevadísimo coste de participación. La información se manifiesta como excluyente y centralizada y, por tanto, contraria a las leyes de la economía liberal. Los datos del estudio ayudan a demostrar tanto la importancia de la economía de la atención, como la vinculación de los usuarios, sobre todo de los estudiantes analizados, en la alimentación de la propia concentración informativa.

El estudio realizado constata que los jóvenes participantes de la investigación tienden a utilizar cada vez más el dispositivo móvil y que su atención se concentra en las redes sociales, especialmente en WhatsApp e Instagram. Los datos de 2019 revelan una tendencia importante de aumento del consumo de redes sociales y los propios estudiantes se declararon adictos a ellas. Sin embargo, ello se produce de forma parcialmente inconsciente. Igualmente inconscientes pero eficaces son los mecanismos que hacen que la persona sienta que debe prestar atención constante a sus dispositivos móviles.

Asimismo, la concentración del mayor tiempo de uso del teléfono móvil en las redes sociales destina y centraliza la intención informativa de los usuarios a los mismos canales, los social media, exentos de verificación y contraste.

El concepto de economía de la atención evoluciona desde referirse a la necesaria selección de la atención que debe hacerse en un escenario de sobreabundancia informativa, hasta ligarse con los mecanismos de control sofisticado que delegan esa selección en algoritmos que actúan bajo la inconsciencia del usuario. De hecho, el estudio demuestra cómo los impulsos emotivos de la muestra participante de la investigación se sitúan por delante de su propia racionalidad.

La investigación pone de manifiesto que la manera de medir la atención del usuario es el tiempo que se dedica a las redes sociales en particular o al teléfono móvil en general, pero además, realiza un aporte con respecto a otros estudios señalados. Mientras otras investigaciones se llevan a cabo, principalmente, a través de encuestas cuantitativas, ésta añade la comparación entre la atención real dedicada al dispositivo móvil y la atención percibida por el usuario. Así, se hace hincapié en la incoherencia entre la cantidad de atención que se cree que se dedica al dispositivo y la que verdaderamente se emplea, lo que pone de re-

La consciencia de la atención como reacción a un estímulo informativo es llevada al extremo bajo la emotividad generada por las notificaciones de las redes sociales 
lieve la inconsciencia del usuario, pero además permite reflexionar sobre ello de forma cualitativa. La variedad de técnicas empleadas en la investigación representa por tanto un avance explicativo del fenómeno analizado. Todo ello representa una evolución hacia la explicación más profunda del problema de investigación como campo de estudio para futuras investigaciones sobre dos líneas de trabajo:

- la exploración longitudinal y comparada del uso del móvil entre estudiantes universitarios de diferentes grados y universidades;

- el análisis de la atención como mercancía principal y como mecanismo de control de la sociedad de la información.

La complejidad del fenómeno se hace patente en el estudio. Los jóvenes usuarios analizados son conscientes tanto del uso excesivo que realizan de las redes sociales y del móvil, como del factor homogeneizador y limitante de ello. Pero aun así, justifican su atención a ellas como "necesaria". Ellos también deciden que "probablemente no quiero cambiar la forma de usar mi teléfono móvil", como indicaba el $41 \%$ de los participantes en el estudio sobre el uso de redes sociales desarrollado por Dscout's en 2016.

\section{Referencias}

Abi-Jaoude, Elia; Treurnicht-Naylor, Karline; Pignatiello, Antonio (2020). "Smartphones, social media use and youth mental health". CMAJ, v. 192, n. 6, pp. 135-141.

https://doi.org/10.1503/cmaj.190434

Ahmed, Niaz (2019). "Generation Z's smartphone and social media usage: A survey". Journalism and mass communication, v. 9, n. 3, pp. 101-122.

https://doi.org/10.17265/2160-6579/2019.03.001

Amador, Norma; Pascual, Sánchez; Hernández, Jorge-Arturo; Guízar, Juan-Manuel (2019). “Principales usos del móvil en estudiantes universitarios de una Facultad de Negocios y su percepción de afectación en la academia". In: Global conference on business and finance proceedings, v. 14, n. 2, pp. 580-585.

Beller, Jonathan (2006). The cinematic mode of production: Attention economy and the society of the spectacle. Lebanon, New Hampshire: University Press of New England. ISBN: 9781584655824

Caliskan, Aylin; Bryson, Joanna J.; Narayanan, Arvind (2017). "Semantics derived automatically from language corpora contain human-like biases". Science, v. 356, n. 6334, pp. 183-186.

https://doi.org/10.1126/science.aal4230

Carcelén, Sonia; Mera, Monstserrat; Irisarri, José-Antonio (2019). “Mobile phone use by university students in Madrid: a management-based typology during times of learning". Communication \& society, v. 32, n. 1, pp. $199-211$. https://doi.org/10.15581/003.32.1.199-211

Celis-Bueno, Claudio (2017). The attention economy. Labour, time and power in cognitive capitalism. Londres: Rowman and Littlefield International. ISBN: 9781783488230

Chou, Hui-Tzu-Grace; Edge, Nicholas (2012). “'They are happier and having better lives than I am': the impact of using Facebook on perceptions of others' lives". Cyberpsychology, behavior, and social networking, v. 15, n. 2, pp. 117-121. http://doi.org/10.1089/cyber.2011.0324

Ciampaglia, Giovanni-Luca; Flammini, Alessandro; Menczer, Filippo (2015). "The production of information in the attention economy". Scientific reports, v. 5, art. 9452.

https://doi.org/10.1038/srep09452

Csikszentmihalyi, Mihaly (1997). Fluir: una psicología de la felicidad. Barcelona: Kairós. ISBN: 9788472453722

Davenport, Thomas H.; Beck, John C. (2002). La economía de la atención: el nuevo valor de los negocios. Barcelona: Paidós. ISBN: 9788449312243

Davis, Stanley M.; Meyer, Christopher (1999). La velocidad de los cambios en la economía interconectada: Blur. Barcelona: Paidós. ISBN: 844930718 X

Del-Val-Cid, Consuelo (2015). “La encuesta con cuestionario estandarizado”. En: Callejo-Gallego, Javier (coord.). Introducción a las técnicas de investigación social. Madrid: Editorial Universitaria Ramón Areces. ISBN: 9788480049320

Deng, Tao; Kanthawala, Shaheen; Meng, Jingbo; Peng, Wei; Kononova, Anastasia; Hao, Qi; Zhang, Qinhao; David, Prabu (2019). "Measuring smartphone usage and task switching with log tracking and self-reports". Mobile media \& communication, v. 7, n. 1, pp. 3-23.

https://doi.org/10.1177/2050157918761491 
Dscout's (2016). Mobile touches. Dscout's inaugural study on humans and their tech. Research report. https://blog.dscout.com/hubfs/downloads/dscout_mobile_touches_study_2016.pdf

Franck, Georg (2002). "The scientific economy of attention: A novel approach to the collective rationality of science". Scientometrics, v. 55, n. 1, pp. 3-26. https://doi.org/10.1023/A:1016059402618

Franck, Georg (2019a). "The economy of attention". Journal of sociology, v. 55, n. 1, pp. 8-19. https://doi.org/10.1177/1440783318811778

Franck, Georg (2019b). "The economy of attention in the age of neoliberalism". In: Doyle, Waddick; Roda, Claudia. Communication in the era of attention scarcity. Cham: Palgrave Macmillan, pp. 33-47. ISBN: 9783030209186 https://doi.org/10.1007/978-3-030-20918-6_4

Fu, Guifang; Yin, Yuting; Guo, Jia (2019). "Mobile phone addiction and career preparation in college students". Higher education of social science, v. 16, n. 2, pp. 10-16. https://doi.org/10.3968/11215

Fuchs, Christian (2012). “Google capitalism”. Triple C. Communication, capitalism \& critique, v. 10, n. 1, pp. 42-48. https://doi.org/10.31269/triplec.v10i1.304

Fuchs, Christian (2014). Social media: a critical introduction. Londres: Sage. ISBN: 9781446257302 https://doi.org/10.4135/9781446270066

Fuchs, Christian (2018). Digital demagogue: Authoritarian capitalism in the age of Trump and Twitter. Londres: Pluto Press. ISBN: 9780745337968

Gerlitz, Carolin; Helmond, Anne (2013). "The like economy: Social buttons and the data-intensive web". New media \& society, v. 15, n. 8, pp. 1348-1365. https://doi.org/10.1177/1461444812472322

Giffard, Alain (2013). "Rhétorique de l'attention et de la lecture". En: Les entretiens du nouveau monde industriel, 17-18 décembre 2012, Centre Pompidou, Paris, pp. 14-44.

https://enmi-conf.org/wp/enmi12

Giraldo-Luque, Santiago (2015). Més enllà de Twitter. De l'expressió indignada a l'acció política. Vic: Eumo. ISBN: 97884 97665537

Giraldo-Luque, Santiago; Tejedor, Santiago; Carniel-Bugs, Ricardo (2017). “Motivaciones de uso de las redes sociales de los estudiantes de periodismo de América Latina y España". Informação \& sociedade: Estudos, v. 27, n. 3, pp. $191-203$. https://doi.org/10.22478/ufpb.1809-4783.2017v27n3.34691

Goldhaber, Michael H. (1997). "The attention economy and the net". First Monday, v. 2, n. 4. https://doi.org/10.5210/fm.v2i4.519

Goldhaber, Michael H. (2006). "The value of openness in an attention economy". First Monday, v. 11, n. 6. https://doi.org/10.5210/fm.v11i6.1334

Gomes-Franco-Silva, Flavia; Sendín-Gutiérrez, José-Carlos (2014). “Internet como refugio y escudo social: usos problemáticos de la Red por jóvenes españoles". Comunicar, n. 43, pp. 45-53.

https://doi.org/10.3916/C43-2014-04

Gower, Aubrey D.; Moreno, Megan A. (2018). "A novel approach to evaluating mobile smartphone screen time for IPhones: feasibility and preliminary findings". JMIR mhealth and uhealth, v. 6, n. 11, pp. 11012.

https://doi.org/10.2196/11012

Guillaud, Hubert (2018). "Internet est en nous...". Sciences humaines, v. 304, n. 6, pp. 5. https://www.cairn.info/magazine-sciences-humaines-2018-6-page-5.htm

Han, Sang-Gyu; Kim, Dong-Tae (2019). "Phenomenological analysis on causes of addiction in smartphone addiction adolescents". Journal of the Korea Convergence Society, v. 10, n. 5, pp. 287-296.

https://doi.org/10.15207/JKCS.2019.10.5.287

Holland, Grace; Tiggemann, Marika (2016) "A systematic review of the impact of the use of social networking sites on body image and disordered eating outcomes". Body image, v. 17, pp. 100-110.

https://doi.org/10.1016/j.bodyim.2016.02.008

Hootsuite; We are social (2019). Global digital 2019 reports.

https://wearesocial.com/global-digital-report-2019 
IAB (2019). Estudio anual de redes sociales 2019. Interactive Advertising Bureau.

https://iabspain.es/wp-content/uploads/2019/06/estudio-anual-redes-sociales-iab-spain-2019_vreducida.pdf

Lewis, Paul (2017). “Our minds can be hijacked: The tech insiders who fear a smartphone dystopia”. The guardian, 6 October. https://www.theguardian.com/technology/2017/oct/05/smartphone-addiction-silicon-valley-dystopia

Luhmann, Niklas (1995). Poder. Barcelona: Anthropos. ISBN: 8476584776

Marcuse, Herbert (1987). El hombre unidimensional. Barcelona: Ariel. ISBN: 8434410222

Mayer-Schönberger, Viktor; Cukier, Kenneth (2013). Big data. La revolución de los datos masivos. Madrid: Turner. ISBN: 9788415832102

Mays, Nicholas; Pope, Catherine (1995). “Qualitative research: Rigour and qualitative research”. Bmj, v. 311, n. 6997, pp. 109-112.

https://doi.org/10.1136/bmj.311.6997.109

Morgans, Julian (2017). "The secret ways social media is built for addiction", Vice, 21 May.

https://www.vice.com/en_uk/article/vv5jkb/the-secret-ways-social-media-is-built-for-addiction

Ofcom (2018). Communications market report 2018. Ofcom.

https://www.ofcom.org.uk/research-and-data/multi-sector-research/cmr/cmr-2018

O'Reilly, Tim (2007). "What is web 2.0: Design patterns and business models for the next generation of software". Communications \& strategies, v. 65, n. 1, pp. 17-37.

https://mpra.ub.uni-muenchen.de/4580

Pariser, Eli (2011). The filter bubble: What the internet is hiding from you. Londres: Penguin. ISBN: 9780141969923

Pasquale, Frank (2015). The black box society: The secret algorithms that control money and information. Cambridge: Harvard University Press. ISBN: 9780674368279

Pasquinelli, Matteo (2009). “Google's page rank algorithm: A diagram of cognitive capitalism and the rentier of the common intellect". In: Becker, Konrad; Stalder, Felix. Deep search: The politics of search beyond Google. Londres: Transaction Publishers, pp: 152-162. ISBN: 9783706547956

https://www.mondotheque.be/wiki/images/3/33/Pasquinelli_Googles_PageRank_Algorithm.pdf

Perrin, Andrew; Kumar, Madhu (2019). "About three-in-ten U.S. adults say they are 'almost constantly' online". Pew Research Center, 25 July.

https://www.pewresearch.org/fact-tank/2019/07/25/americans-going-online-almost-constantly

Przybylski, Andrew K.; Murayama, Kou; DeHaan Cody R.; Gladwell, Valerie (2013). “Motivational, emotional and behavioral correlates of fear of missing out". Computers in human behavior, v. 29, n. 4, pp. 1841-1848.

https://doi.org/10.1016/j.chb.2013.02.014

Rahwan, Iyad (2018). "Society-in-the-loop: Programming the algorithmic social contract". Ethics and information technology, v. 20, n. 5, pp. 5-14.

https://doi.org/10.1007/s10676-017-9430-8

Rastreator (2019). “Más de 7,6 millones de españoles se consideran adictos al móvil”. Rastreator, 10 julio.

https://www.rastreator.com/sala-de-prensa/notas-de-prensa/2018-07-adiccion-movil-mas-de-siete-millones-adictos.aspx

Rather, Mudasir-Khazer; Rather, Shabir-Ahmad (2019) “Impact of smartphones on young generation". Library philosophy and practice, n. 2384.

https://digitalcommons.unl.edu/libphilprac/2384

Roda, Claudia (2019). "A roadmap of studies in attention and digital technology". In: Doyle, Waddick; Roda, Claudia. Communication in the era of attention scarcity. Cham: Palgrave Macmillan, pp. 7-20.

https://doi.org/10.1007/978-3-030-20918-6_2

Romero-Rodríguez, José-María; Aznar-Díaz, Inmaculada (2019). “Análisis de la adicción al smartphone en estudiantes universitarios. Factores influyentes y correlación con la autoestima”. RED. Revista de educación a distancia, v. 19 , n. 60. https://doi.org/10.6018/red/60/08

Ruiz-Palmero, Julio; Sánchez-Rivas, Enrique; Gómez-García, Melchor; Sánchez-Vega, Susana (2019). “Future teachers' smartphone uses and dependence". Education sciences, v. 9, n. 3, pp. 194-201.

https://doi.org/10.3390/educsci9030194

Sampasa-Kanyinga, Hugues; Roumeliotis, Paul; Xu, Hao (2014). "Associations between cyberbullying and school bullying victimization and suicidal ideation, plans and attempts among Canadian schoolchildren". PLoS one, v. 9, n, 7, e102145.

https://doi.org/10.1371/journal.pone.0102145 
Shapiro, Carl; Varian, Hal R. (1999). Information rules: A strategic guide to the network economy. Boston: Harvard Business Press. ISBN: $087584863 \mathrm{X}$

Simon, Herbert A. (1971). "Designing organizations for an information-rich world”. In: Greenberger, M. (ed.). Computers, communications and the public interest. ISBN: 080181135X

https://digitalcollections.library.cmu.edu/awweb/awarchive?type=file\&item $=33748$

Sunstein, Cass R. (2017). \#Republic: divided democracy in the age of social media. Princeton and Oxford: Princeton University Press. ISBN: 9780691180908

Tejedor, Santiago; Carniel-Bugs, Ricardo; Giraldo-Luque, Santiago (2019). “Millennials e internet: cómo los estudiantes de comunicación iberoamericanos utilizan y valoran las redes sociales". Anàlisi, n. 60, pp. 43-63.

https://doi.org/10.5565/rev/analisi.3167

Turkle, Sherry (2017). En defensa de la conversación. El poder de la conversación en la era digital. Barcelona: Ático. ISBN: 9788416222278

Vempati, Srimanth; Bhuma, Manoj-Kumar; Fiaidhi, Jinan (2020). “Fear of missing out, social media engagement, smartphone addiction and distraction moderating role of tracking apps in the youth". TechRxiv. Preprint.

https://doi.org/10.36227/techrxiv.12094107.v1

Wise, J. Macgregor (2012). "Attention and assemblage in the clickable world". In: Packer, Jeremy; Crofts-Wiley, Stephen B. Communication matters: materialist approaches to media, mobility and networks. Oxon: Routledge, pp. 159-172. ISBN: 9780415782241

https://www.routledge.com/Communication-Matters-Materialist-Approaches-to-Media-Mobility-and-Networks/ Packer-B-Crofts-Wiley-Zelizer/p/book/9780415782258

Žižek, Slavoj (2018). El coraje de la desesperanza. Crónicas del año en que actuamos peligrosamente. Barcelona: Anagrama. ISBN: 9788433964267

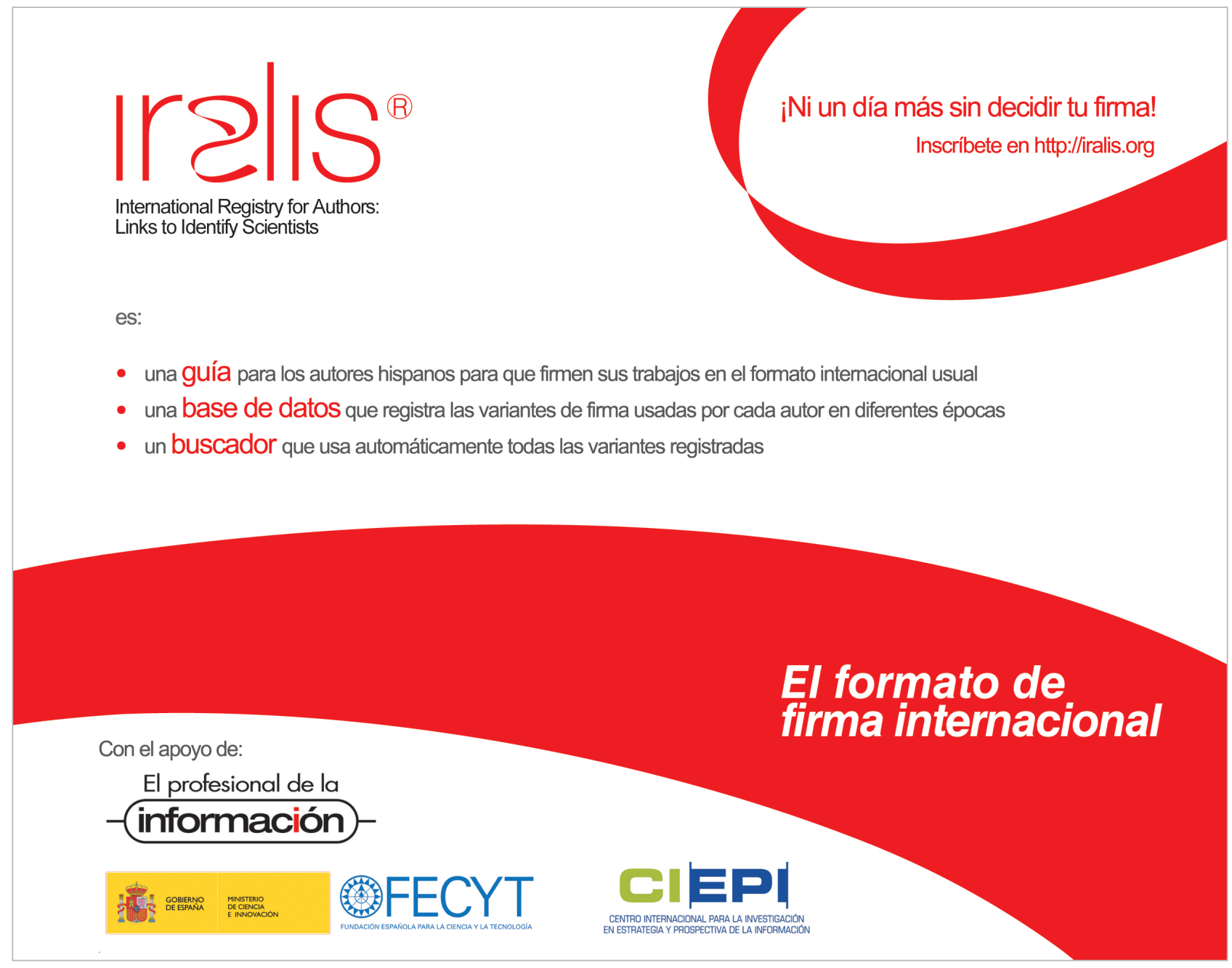

